\title{
A NEW CLASS OF CONTINUED FRACTION EXPANSIONS FOR THE RATIOS OF HEINE FUNCTIONS. II $\left({ }^{1}\right)$
}

\author{
BY \\ EVELYN FRANK
}

\begin{abstract}
Dedicated to Professor Oskar Perron on the occasion of his eightieth birthday, May 7, 1960.
\end{abstract}

1. Introduction. New continued fraction expansions for the ratios of two contiguous Heine functions $[3 ; 4 ; 5]\left({ }^{2}\right)$ are described here in detail $(\$ 2,3)$. The characterizing feature of each of these expansions is the fact that it is equal throughout the finite $z$-plane to the ratio of the functions which generates it. It is equal to this ratio in the neighborhood of the origin and furnishes the analytic continuation of this ratio throughout the finite $z$-plane. In sharp contrast to the continued fraction expansions investigated here are those previously found by the author [1] for the ratios of contiguous Heine functions. Each of the latter expansions converges inside a certain circular region about the origin, dependent on the values of $a, b, c$, and $q$, to the ratio of the two Heine functions which generates the expansion; exterior to this circular region, each expansion converges to the ratio of two different Heine functions.

The Heine function $\left({ }^{3}\right)$ is the infinite series

$$
\begin{aligned}
\phi(a, b, c, q, z)=1 & +\frac{\left(1-q^{a}\right)\left(1-q^{b}\right)}{(1-q)\left(1-q^{c}\right)} z \\
& +\frac{\left(1-q^{a}\right)\left(1-q^{a+1}\right)\left(1-q^{b}\right)\left(1-q^{b+1}\right)}{(1-q)\left(1-q^{2}\right)\left(1-q^{c}\right)\left(1-q^{c+1}\right)} z^{2}+\cdots
\end{aligned}
$$

with radius of convergence equal to 1 if $|q|<1$ and equal to $\left|q^{c+1-a-b}\right|$ if $|q|>1$. In (1.1), it is understood that $|q| \neq 1, c \neq \neq-p \pm 2 n \pi i / u$, where $q=e^{u}, n, p=0,1,2, \cdots$. Except for these conditions, $a, b, c$, and $q$ are arbitrary. If $a$ or $b$ is equal to $-p \pm 2 n \pi i / u, \phi(a, b, c, q, z)$ reduces to a polynomial.

In $\$ 4$ certain equal continued fractions are studied. They are equal as consequences of the expansions found in [1] and the entirely different types found in $\S \S 2$ and 3 here, since both types converge to the same ratios of contiguous Heine functions. In $\S \S 5$ special continued fraction expansions obtained from $\$ \S 2$ and 3 are considered.

Presented to the Society, August 26, 1958; received by the editors April 8, 1959.

(1) Research sponsored by the Office of Ordnance Research, U. S. Army, under Contract No. DA-11-022 ORD-2196 (Supplemental).

(2) Numbers in brackets refer to the bibliography at the end of the paper.

(3) Heine (cf. $[3 ; 4]$ ) used, for example, $q^{a}$, which is not single valued. However, by the use of $e^{\text {ua }}$, which is single valued, one does not need to specify the branch of $q^{a}$. For simplification in printing, the notation $q^{a}$ (with the understanding that $e^{\text {ua }}$ is meant) is used throughout here (cf. also [6]). 
2. The expansions for $\phi(a, b, c, q, z): \phi(a+1, b+1, c+1, q, z)$. The continued fraction expansion $\left({ }^{4}\right)$

$$
\begin{aligned}
& \frac{\phi(a, b, c, q, z)}{\phi(a+1, b+1, c+1, q, z)} \sim b_{0}+\frac{a_{1}}{b_{1}}+\frac{a_{2}}{b_{2}}+\cdots, \\
& a_{p+1}=\frac{\left(1-q^{a+p+1}\right)\left(1-q^{b+p+1}\right)\left(q^{c+p} z-q^{a+b+2 p+1} z^{2}\right)}{\left(1-q^{c+p+1}\right)\left(1-q^{c+p}\right)}, \\
& b_{p}=1-\frac{\left(q^{a+p}+q^{b+p}-q^{a+b+2 p}-q^{a+b+2 p+1}\right) z}{1-q^{c+p}}, \quad p=0,1,2, \cdots,
\end{aligned}
$$

is obtained by successive substitution of the Heine identity [4, p. 292]

$$
\begin{aligned}
& \left(1-q^{c}\right)\left(1-q^{c+1}\right) \phi(a, b, c, q, z) \\
& -\left(1-q^{c+1}\right)\left[1-q^{c}-\left(q^{a}+q^{b}-q^{a+b}-q^{a+b+1}\right) z\right] \\
& \quad \cdot \phi(a+1, b+1, c+1, q, z)-q^{c} z\left(1-q^{a+1}\right)\left(1-q^{b+1}\right) \cdot\left(1-q^{a+b+1-c} z\right) \\
& \quad \cdot \phi(a+2, b+2, c+2, q, z)=0 .
\end{aligned}
$$

Expansion (2.1) is analogous to expansion (4.4) of [2] for the ratio of the corresponding Gauss functions.

For the determination of the convergence $\left({ }^{5}\right)$, one notes that $(2.1)$ is limitperiodic (cf. [1]). If $|q|<1$ in (2.1), $\lim _{p \rightarrow \infty} a_{p}=0, \lim _{p \rightarrow \infty} b_{p}=1$ uniformly in any bounded region $0 \leqq|z| \leqq C_{1}$, and the roots of the auxiliary equation $x^{2}=\lim _{p \rightarrow \infty} b_{p} \cdot x+\lim _{p \rightarrow \infty} a_{p}$ are $\rho_{1}=1, \rho_{2}=0$. Furthermore, there exist positive numbers $\theta<1, c$, and $C$, such that in the entire region $c \leqq\left|\rho_{1}\right| \leqq C$, $\left|\rho_{2} / \rho_{1}\right| \leqq \theta$. (Here, $\theta=0, c=C=1$, satisfy these conditions.) Then, by Theorem 2.42 of Perron [6, p. 93], there exists a number $n$ such that, for $p \geqq n$, the continued fraction

$$
b_{p}+\frac{a_{p+1}}{b_{p+1}}+\frac{a_{p+2}}{b_{p+2}}+\cdots
$$

converges uniformly in the entire region $0 \leqq|z| \leqq C_{1}$. Now, by a proof analogous to that of Perron [6, p. 146, Theorem 3.24], since (2.3) converges uniformly if $|q|<1,(2.1)$ is equal to the corresponding power series in the neighborhood of the origin, and furnishes the analytic continuation through-

(4) The symbol $\sim$ denotes a formal expansion. If at any time a partial numerator vanishes while the corresponding partial denominator does not vanish, the continued fraction breaks off with the preceding term. In this case the symbol $\sim$ can be replaced by the $=$ sign.

(5) In any of the expansions in $\$ 2$ and 3 , for certain values of $a, b$, or $c$, a partial numerator of a continued fraction may be zero. In this case, the continued fraction is finite, and its value can be computed therefrom. Furthermore, it is understood throughout that in each case those values of $a, b$, or $c$ are excluded which make indeterminate the function to which the continued fraction in question converges. 
out the finite $z$-plane. Thus, if $|q|<1,(2.1)$ holds with the equality sign replacing the $\sim$ sign.

For the consideration of the case $|q|>1$, let $q$ and $z$ be replaced by $1 / q$ and $z q^{a+b-c}$, respectively, in (2.1). Then, with the use of the formula $\phi\left(a, b, c, 1 / q, z q^{a+b-c}\right)=\phi(a, b, c, q, q z)$ and of the Heine identity [4, p. 287]

$$
\phi(a, b, c, q, z)-\phi(a, b, c, q, q z)
$$

$$
=\frac{\left(1-q^{a}\right)\left(1-q^{b}\right)}{1-q^{c}} z \cdot \phi(a+1, b+1, c+1, q, z),
$$

from (2.1) one obtains

$$
\begin{aligned}
& \frac{\phi(a, b, c, q, z)}{\phi(a+1, b+1, c+1, q, z)} \sim \frac{\left(1-q^{a}\right)\left(1-q^{b}\right)}{1-q^{c}} z+b_{0}+\frac{a_{1}}{b_{1}}+\frac{a_{2}}{b_{2}}+\cdots, \\
& a_{p+1}=\frac{\left(1-q^{a+p+1}\right)\left(1-q^{b+p+1}\right)\left(z / q^{p+1}-z^{2} / q^{2 p+2}\right)}{\left(1-q^{c+p+1}\right)\left(1-q^{c+p}\right)}, \\
& b_{p}=1+\frac{\left(q^{b+p}+q^{a+p}-1-q^{-1}\right) \cdot z / q^{p}}{1-q^{c+p}}, \quad p=0,1,2, \cdots .
\end{aligned}
$$

If $|q|>1, \lim _{p \rightarrow \infty} a_{p}=0, \lim _{p \rightarrow \infty} b_{p}=1$ in (2.5), and the roots of the auxiliary equation are again unequal. By the same argument used in the preceding case, it follows that, if $|q|>1,(2.5)$ converges throughout the finite $z$-plane to the function $\phi(a, b, c, q, z): \phi(a+1, b+1, c+1, q, z)$, and the following theorem has been proved.

THEOREM 2.1. If $|q|<1$, the continued fraction (2.1) represents a meromorphic function of $z$ which is equal to the function $\phi(a, b, c, q, z)$ : $\phi(a+1, b+1, c+1, q, z)$ throughout the finite $z$-plane. It is equal to this ratio in the neighborhood of the origin and furnishes the analytic continuation of it throughout the finite z-plane. If $|q|>1$, a similar statement holds for the continued fraction (2.5).

3. Other continued fraction expansions for the ratios of contiguous Heine functions. One obtains the continued fraction expansion

$$
\begin{aligned}
& \frac{\phi(a, b, c, q, z)}{\phi(a, b, c+1, q, z)} \sim \frac{1}{1-q^{a+b-c-1} z} \cdot\left[b_{0}+\frac{a_{1}}{b_{1}}+\frac{a_{2}}{b_{2}}+\cdots\right], \\
& a_{p+1}=\frac{q^{a+b-c-p-2} z\left(1-q^{c-a+p+1}\right)\left(1-q^{c-b+p+1}\right)\left(1-q^{a+b-c-p-2} z\right)}{\left(1-q^{c+p+1}\right)\left(1-q^{c+p}\right)}, \\
& b_{p}=1-\frac{\left(q^{a+b-c-p-1}+q^{a+b-c-p-2}-q^{a-1}-q^{b-1}\right) z}{1-q^{c+p}}, \quad p=0,1, \cdots,
\end{aligned}
$$

by successive substitution in the Heine identity [4, p. 290] 


$$
\begin{aligned}
\left(1-q^{c+1}\right)[ & \left.1-q^{c}+z\left(q^{a-1}+q^{b-1}-q^{a+b-c-2}-q^{a+b-c-1}\right)\right\rfloor \\
& \cdot \phi(a, b, c+1, q, z)+q^{a+b-c-2} z\left(1-q^{c-a+1}\right)\left(1-q^{c-b+1}\right) \\
& \cdot \phi(a, b, c+2, q, z)-\left(1-q^{c}\right)\left(1-q^{c+1}\right)\left(1-q^{a+b-c-1} z\right) \\
& \cdot \phi(a, b, c, q, z)=0
\end{aligned}
$$

and by an equivalence transformation.

For the determination of the convergence of (3.1), if $|q|>1, \lim _{p \rightarrow \infty} a_{p}=0$, $\lim _{p \rightarrow \infty} b_{p}=1$, and the roots of the auxiliary equation are 1 and 0 . By the same proof as for Theorem 2.1, it can be proved that the continued fraction (3.1) converges throughout the finite $z$-plane to the function $\phi(a, b, c, q, z)$ : $\phi(a, b, c+1, q, z)$ if $|q|>1$.

For the consideration of the case $|q|<1$, let $q$ and $z$ be replaced by $1 / q$ and $z q^{a+b-c-1}$, respectively, in the expansion (3.1). Then, with the use of the formulas $\phi\left(a, b, c, 1 / q, z q^{a+b-c-1}\right)=\phi(a, b, c, q, z)$ and $\phi\left(a, b, c+1,1 / q, z q^{a+b-c-1}\right)$ $=\phi(a, b, c+1, q, q z)$, one obtains the continued fraction expansion for $(1-z) \cdot \phi(a, b, c, q, z): \phi(a, b, c+1, q, q z)$. Finally, with the use of the identity (2.4) and the Heine identity [4, p. 292]

$$
\begin{aligned}
\phi(a, b, c, q, z)- & \phi(a, b, c+1, q, z) \\
= & q^{c} z \cdot \frac{\left(1-q^{a}\right)\left(1-q^{b}\right)}{\left(1-q^{c}\right)\left(1-q^{c+1}\right)} \cdot \phi(a+1, b+1, c+2, q, z),
\end{aligned}
$$

one obtains the continued fraction expansion

$$
\begin{aligned}
& \frac{\phi(a, b, c, q, z)}{\phi(a, b, c+1, q, z)}=\frac{1}{1-q^{c}}+\frac{q^{c}(1-z)}{b_{0}}+\frac{a_{1}}{b_{1}}+\frac{a_{2}}{b_{2}}+\cdots, \\
& a_{p+1}=\frac{q^{a+b+p} z\left(1-q^{c-a+p+1}\right)\left(1-q^{c-b+p+1}\right)\left(1-q^{p+1} z\right)}{\left(1-q^{c+p+1}\right)\left(1-q^{c+p}\right)}, \\
& b_{p}=1-\frac{\left(q^{b+p}+q^{a+p}-q^{c+2 p+1}-q^{c+2 p}\right) z}{1-q^{c+p}}, p=0,1, \cdots,|q|<1 .
\end{aligned}
$$

The equality sign holds in (3.4) for $|q|<1$ since this formula was obtained from (3.1) by the replacement of $q$ and $z$ by $1 / q$ and $z q^{a+b-c-1}$, respectively, and it has just been shown that the equality sign holds in (3.1) if $|q|>1$. Consequently, one has the following theorem.

THEOREM 3.1. The continued fraction (3.1) represents a meromorphic function of $z$ which is equal to the function $\phi(a, b, c, q, z): \phi(a, b, c+1, q, z)$ throughout the finite z-plane if $|q|>1$. It is equal to this ratio in the neighborhood of the origin and furnishes the analytic continuation of it throughout the finite z-plane. A similar statement holds for the continued fraction (3.4) if $|q|<1$. 
It is remarked that if $|q|<1$, one can obtain a continued fraction expansion for the ratio $\phi(a, b, c, q, z): \phi(a, b, c+1, q, z)$ by use of the expansion (2.1) and the identity

$$
\begin{aligned}
& -\left(1-q^{a+b-c-1} z \cdot \phi(a, b, c, q, z)+\phi(a-1, b-1, c, q, z)\right. \\
& -\frac{q^{a+b-c-1} z}{1-q^{c}}\left(1+q^{c-1}-q^{c-a}-q^{c-b}\right) \phi(a, b, c+1, q, z)=0,
\end{aligned}
$$

which is obtained from the Heine identities [4, p. 291, formulas (33) and (34)]. It is as follows:

$$
\begin{aligned}
& \frac{\phi(a, b, c, q, z)}{\phi(a, b, c+1, q, z)}=1+\frac{1}{1-q^{a+b-c-1} z}\left[\frac{a_{1}}{b_{1}}+\frac{a_{2}}{b_{2}}+\cdots\right] \\
& a_{p}=\frac{\left(1-q^{a+p-1}\right)\left(1-q^{b+p-1}\right)\left(q^{c+p-1} z-q^{a+b+2 p-3} z^{2}\right)}{\left(1-q^{c+p}\right)\left(1-q^{c+p-1}\right)}, \\
& b_{p}=1-\frac{\left(q^{a+p-1}+q^{b+p-1}-q^{a+b+2 p-2}-q^{a+b+2 p-1}\right) z}{1-q^{c+p}}, \\
& p=1,2, \cdots,|q|<1 .
\end{aligned}
$$

A number of other expansions for the ratios of contiguous Heine functions can be obtained from (2.1), (2.5), (3.1), (3.4), and (3.6). For example, the continued fraction expansion

$$
\begin{aligned}
& \frac{\phi(a, b, c, q, z)}{\phi(a+1, b, c, q, z)}=1-\frac{q^{a} z\left(1-q^{b}\right)}{1-q^{c-a-1}}+\frac{1-q^{c-1}}{1-q^{c-a-1}}\left[\frac{a_{1}}{b_{1}}+\frac{a_{2}}{b_{2}}+\cdots\right], \\
& a_{p}=\frac{\left(1-q^{a+p}\right)\left(1-q^{b+p-1}\right)\left(q^{c+p-2} z-q^{a+b+2 p-2} z^{2}\right)}{\left(1-q^{c+p-1}\right)\left(1-q^{c+p-2}\right)} \\
& b_{p}=1-\frac{\left(q^{a+p}+q^{b+p-1}-q^{a+b+2 p-1}-q^{a+b+2 p}\right) z}{1-q^{c+p-1}}, \\
& \quad p=1,2, \cdots,|q|<1,
\end{aligned}
$$

is derived from the expansion (2.1) and from the identity

$$
\begin{aligned}
\left(1-q^{c-1}\right) \phi(a, b- & 1, c-1, q, z)-\left(1-q^{c-a-1}\right) \phi(a, b, c, q, z) \\
& -q^{c-a-1}\left(1-q^{a}\right)\left(1-q^{a+b-c} z\right) \phi(a+1, b, c, q, z)=0,
\end{aligned}
$$

which is obtained from the Heine identities [4, p. 291, formulas (34) and (35)].

Since the Heine function is symmetric in $a$ and $b$, on interchanging $a$ and $b$ in (3.7), one obtains the expansion for $\phi(a, b, c, q, z): \phi(a, b+1, c, q, z)$.

Similarly, from (2.5) and (3.8) one obtains the expansion 


$$
\begin{gathered}
\frac{\phi(a, b, c, q, z)}{\phi(a+1, b, c, q, z)}=1-\frac{q^{-1} z\left(1-q^{b}\right)}{1-q^{c-a-1}}+\frac{1-q^{c}}{1-q^{c-a-1}}\left[\frac{a_{1}}{b_{1}}+\frac{a_{2}}{b_{2}}+\ldots\right], \\
\text { (3.9) } a_{p}=\frac{\left(1-q^{a+p}\right)\left(1-q^{b+p-1}\right)\left(z / q^{p}-z^{2} / q^{2 p}\right)}{\left(1-q^{c+p-1}\right)\left(1-q^{c+p}\right)}, \\
b_{p}=1+\frac{\left(q^{a+p}+q^{b+p-1}-1-q^{-1}\right) \cdot z / q^{p}}{1-q^{c+p-1}}, \quad p=1,2, \cdots,|q|>1 .
\end{gathered}
$$

From the numerous Heine identities, one can in a similar way obtain continued fraction expansions for many other ratios of contiguous Heine functions. To illustrate, from the following identity, one can write the continued fraction expansions for the ratio in the left-hand member on substitution of the appropriate expansions derived from (3.7) and (3.9) in the righthand member:

$$
\frac{\phi(a, b, c, q, z)}{\phi(a, b+1, c+1, q, z)}=\frac{q^{c-b}\left(1-q^{b}\right)}{1-q^{c}}+\frac{1-q^{c-b}}{1-q^{c}} \cdot \frac{\phi(a, b, c+1, q, z)}{\phi(a, b+1, c+1, q, z)} .
$$

4. Equal continued fractions $\left({ }^{6}\right)$. Since certain expansions in [1] and in $\S \S 2$ and 3 above converge to the same generating ratios in common domains, one obtains the following theorems on equal continued fractions. In particular, from the well-known continued fraction of Heine (cf. $[3 ; 4 ; 5])$, from (3.10) above, and from expansion (2.1) of [1], one obtains the equal continued fractions in (4.1).

TheORem 4.1. If $|q| \neq 1$,

$$
\begin{aligned}
& \frac{q^{a-1} z\left(1-q^{b}\right)}{1-q^{c}}+b_{0}+\frac{a_{1}}{b_{1}}+\frac{a_{2}}{b_{2}}+\cdots=1+\frac{d_{1}}{f_{1}+1}+\frac{d_{2}}{f_{2}+1}+\cdots, \\
& \left.=1+\frac{e_{1}}{1}+\frac{e_{2}}{1}+\cdots=q^{b+a+2 p} z^{2}\right) \\
& a_{p+1}=\frac{\left(1-q^{b+p+1}\right)\left(1-q^{a+p}\right)\left(q^{c+p} z-q^{b+a}\right.}{\left(1-q^{c+p}\right)\left(1-q^{c+p+1}\right)} \\
& b_{p}=1-\frac{\left(q^{b+p}+q^{a+p-1}-q^{b+a+2 p-1}-q^{b+a+2 p}\right) z}{1-q^{c+p}}, \\
& \quad p=0,1, \cdots,|q|<1,
\end{aligned}
$$

or

${ }^{(6)}$ It is understood throughout this section concerning equal continued fractions that (i) if a partial numerator is zero, the continued fraction breaks off with the preceding term; (ii) both continued fractions can diverge (if they are $\infty$ ), or, if one of the continued fractions is indeterminate, the other diverges unless it is finite, in which case it is also indeterminate. 


$$
\begin{array}{ll}
a_{p}=\frac{\left(1-q^{a+p-1}\right)\left(1-q^{b+p}\right)\left(z / q^{p}-z^{2} / q^{2 p}\right)}{\left(1-q^{c+p}\right)\left(1-q^{c+p-1}\right)}, & b_{0}=1-\frac{q^{-1} z\left(1-q^{a+b}\right)}{1-q^{c}}, \\
b_{p}=1+\frac{\left(q^{b+p}+q^{a+p-1}-1-q^{-1}\right) \cdot z / q^{p}}{1-q^{c+p}}, & p=1,2, \cdots,|q|>1,
\end{array}
$$

(4.1) $e_{2 p-1}=-q^{b+p-1} z \cdot \frac{\left(1-q^{a+p-1}\right)\left(1-q^{c-b+p-1}\right)}{\left(1-q^{c+2 p-2}\right)\left(1-q^{c+2 p-1}\right)}$,

$$
\begin{array}{cc}
e_{2 p}=-q^{a+p-1} z \cdot \frac{\left(1-q^{b+p}\right)\left(1-q^{c-a+p}\right)}{\left(1-q^{c+2 p-1}\right)\left(1-q^{c+2 p}\right)}, & p=1,2, \cdots, \\
d_{p}=-q^{b} z \cdot \frac{\left(1-q^{a+p-1}\right)\left(1-q^{c+p-1-b}\right)}{\left(1-q^{c+p-1}\right)\left(1-q^{c+p}\right)}, & f_{p}=q^{b z} \cdot \frac{1-q^{a+p-1-b}}{1-q^{c+p}}, \\
p=1,2, \cdots .
\end{array}
$$

The third member of the equality is equal to the first two only for values of $z$ such that $|z|<\left|q^{-b}\right|$ if $|q|<1$, and $|z|<\left|q^{c+1-a}\right|$ if $|q|>1$.

From (4.1) the following special cases are obtained, where the equality signs hold only for those values of $z$ specified in Theorem 4.1.

For $b-c=n=0,1, \cdots$, in (4.1), the following finite continued fractions are found equal to an infinite one,

$$
\begin{aligned}
\frac{q^{a-1} z\left(1-q^{c+n}\right)}{1-q^{c}} & +b_{0}+\frac{a_{1}}{b_{1}}+\frac{a_{2}}{b_{2}}+\cdots \\
= & 1+\frac{e_{1}}{1}+\cdots+\frac{e_{2 n}}{1}=1+\frac{d_{1}}{f_{1}+1}+\cdots+\frac{d_{n}}{f_{n}+1},
\end{aligned}
$$

where the $a_{p}, b_{p}, e_{p}, d_{p}$, and $f_{p}$ are given in (4.1) with $b$ replaced by $c+n$.

For $n=1$ in (4.2),

$$
\frac{q^{a-1} z\left(1-q^{c+1}\right)}{1-q^{c}}+b_{0}+\frac{a_{1}}{b_{1}}+\frac{a_{2}}{b_{2}}+\cdots
$$

$$
=1-\frac{\frac{q^{c+1} z\left(1-q^{-1}\right)\left(1-q^{a}\right)}{\left(1-q^{c}\right)\left(1-q^{c+1}\right)}}{1}-\frac{q^{a} z \cdot \frac{1-q^{c-a+1}}{1-q^{c+1}}}{1},
$$

where the $a_{p}$ and $b_{p}$ are given in (4.1) with $b=c+1$.

For $a=c+1, b=c+1$, in (4.1),

$$
\frac{q^{c} z\left(1-q^{c+1}\right)}{1-q^{c}}+b_{0}+\frac{a_{1}}{b_{1}}+\frac{a_{2}}{b_{2}}+\cdots=1-\frac{q^{c+1} z\left(1-q^{-1}\right)}{1-q^{c}} .
$$

For $b=c$ in (4.1), one obtains 


$$
q^{a-1} z+b_{0}+\frac{a_{1}}{b_{1}}+\frac{a_{2}}{b_{2}}+\cdots=1 .
$$

For $a=b=c$ in $(4.1)$,

$$
\begin{gathered}
q^{c-1} z+b_{0}+\frac{a_{1}}{b_{1}}+\frac{a_{2}}{b_{2}}+\cdots=1, \\
a_{p+1}=q^{c+p} z\left(1-q^{c+p} z\right), \quad b_{p}=1-q^{c+p} z\left(1+q^{-1}\right), \\
p=0,1, \cdots,|q|<1 ; \\
a_{p}=\left(1-z / q^{p}\right) z / q^{p}, \quad b_{0}=1-q^{-1} z\left(1+q^{c}\right), \quad b_{p}=1-\left(1+q^{-1}\right) z / q^{p}, \\
p=1,2, \cdots,|q|>1 .
\end{gathered}
$$

It is remarked that the equalities of the first and third members in formula (4.1) can also be obtained when one equates other corresponding infinite continued fractions of [1] and of $\$ \S 2$ and 3 above.

Since continued fractions (3.1) and (3.6) above, and (2.7) of [1], converge to the same ratio of Heine functions, the following theorem holds.

THEOREM 4.2. For values of $z$ such that $|z|<\left|q^{-b}\right|$ if $|q|<1$, and $|z|$ $<\left|q^{c+1-a}\right|$ if $|q|>1$,

$$
\begin{aligned}
& b_{0}+\frac{a_{1}}{b_{1}}+\frac{a_{2}}{b_{2}}+\cdots=1+\frac{d_{1}}{f_{1}+1}+\frac{d_{2}}{f_{2}+1}+\cdots, \\
& b_{0}=\frac{1-q^{c}+z\left(q^{a-1}+q^{b-1}-q^{a+b-c-2}-q^{a+b-c-1}\right)}{\left(1-q^{c}\right)\left(1-q^{a+b-c-1} z\right)}, \\
& a_{1}=\frac{q^{a+b-c-2} z\left(1-q^{c-a+1}\right)\left(1-q^{c-b+1}\right)\left(1-q^{a+b-c-2} z\right)}{\left(1-q^{c+1}\right)\left(1-q^{c}\right)\left(1-q^{a+b-c-1} z\right)}
\end{aligned}
$$

and where the $a_{p+1}, b_{p}, p=1,2, \cdots$, are given in (3.1) if $|q|>1$; or, if $|q|<1$,

$$
b_{0}=1, \quad a_{1}=\frac{\left(1-q^{a}\right)\left(1-q^{b}\right)\left(q^{c} z-q^{a+b-1} z^{2}\right)}{\left(1-q^{c}\right)\left(1-q^{c+1}\right)\left(1-q^{a+b-c-1} z\right)},
$$

and where the $a_{p+1}, b_{p}$, are given in (3.6);

$$
\begin{aligned}
d_{1} & =q^{c} z \cdot \frac{\left(1-q^{a}\right)\left(1-q^{b}\right)}{\left(1-q^{c}\right)\left(1-q^{c+1}\right)}, & d_{p} & =-q^{b} z \cdot \frac{\left(1-q^{a+p-1}\right)\left(1-q^{c+p-1-b}\right)}{\left(1-q^{c+p-1}\right)\left(1-q^{c+p}\right)}, \\
f_{1} & =-q^{a} z \cdot \frac{1-q^{b}}{1-q^{c+1}}, & f_{p} & =q^{b} z \cdot \frac{1-q^{a+p-1-b}}{1-q^{c+p}}, \quad p=2,3, \cdots .
\end{aligned}
$$

For $b-c=n$, and $|q|<1$ in (4.7), one obtains an infinite continued fraction equal to a finite one (in the right-hand member). For $b-c=1,|q|<1$, formula (4.7) reduces to $(4.5)$. 
5. Special continued fractions. From expansion (3.7) one obtains as a special case

$$
\begin{array}{ll}
\frac{1}{\phi(a, 1, c, q, z)}=b_{0}+\frac{a_{1}}{b_{1}}+\frac{a_{2}}{b_{2}}+\cdots, & |q|<1, \\
a_{p+1}=\frac{q^{c+p-1} z\left(1-q^{p+1}\right)\left(1-q^{a+p}\right)\left(1-q^{a+p+1-c} z\right)}{\left(1-q^{c+p-1}\right)\left(1-q^{c+p}\right)} & \\
b_{p}=1-\frac{q^{p} z\left(1+q^{a-1}-q^{a+p-1}-q^{a+p}\right)}{1-q^{c+p-1}}, \quad p=0,1, \cdots .
\end{array}
$$

A different type of continued fraction expansion for this function is shown in $[1$, formula (4.1)]. Here

$$
\phi(a, 1, c, q, z)=1+\frac{1-q^{a}}{1-q^{c}} z+\frac{\left(1-q^{a}\right)\left(1-q^{a+1}\right)}{\left(1-q^{c}\right)\left(1-q^{c+1}\right)} z^{2}+\cdots .
$$

For $c=1$, (5.1) becomes

$$
\begin{array}{ll}
\frac{1}{\phi(a, 1,1, q, z)}=b_{0}+\frac{a_{1}}{b_{1}}+\frac{a_{2}}{b_{2}}+\cdots, & |q|<1, \\
a_{p+1}=q^{p} z \frac{\left(1-q^{a+p}\right)\left(1-q^{a+p}\right)}{1-q^{p}}, & \\
b_{p}=1-\frac{q^{p} z\left(1+q^{a-1}-q^{a+p-1}-q^{a+p}\right)}{1-q^{p}}, & p=0,1, \cdots .
\end{array}
$$

(cf. [1, formula (4.2)]). This is a continued fraction expansion for the reciprocal of

$$
\begin{aligned}
\phi(a, 1,1, q, z) & =1+\frac{1-q^{a}}{1-q} z+\frac{\left(1-q^{a}\right)\left(1-q^{a+1}\right)}{(1-q)\left(1-q^{2}\right)} z^{2}+\cdots \\
& =\prod_{p=0}^{\infty} \frac{1-q^{a+p_{z}}}{1-q^{p_{z}}}
\end{aligned}
$$

(cf. [5, p. 99] and $[4$, p. 303]).

The above formulas also hold with $a$ and $b$ interchanged.

For the reciprocal of

$$
\frac{\phi(1,1,2, q, z)}{1-q}=\frac{1}{1-q}+\frac{z}{1-q^{2}}+\frac{z^{2}}{1-q^{3}}+\cdots .
$$

[5, p. 99], one obtains the expansion 


$$
\begin{gathered}
\frac{1-q}{\phi(1,1,2, q, z)}=(1-q)\left[b_{0}+\frac{a_{1}}{b_{1}}+\frac{a_{2}}{b_{2}}+\ldots\right], \quad|q|<1, \\
a_{p-1}=\frac{q^{p+1} z\left(1-q^{p+1}\right)\left(1-q^{p} z\right)}{1-q^{p+2}}, \quad b_{p}=1-q^{p} z\left(1+\frac{1-q^{p}}{1-q^{p+1}}\right) \\
p=0,1, \cdots .
\end{gathered}
$$

A different type of expansion for this ratio is found in [1, formula (4.3)].

For the reciprocal of

$$
\frac{\phi\left(1,1 / 2,3 / 2, q^{2}, z^{2}\right)}{1-q}=\frac{1}{1-q}+\frac{z^{2}}{1-q^{3}}+\frac{z^{4}}{1-q^{5}}+\cdots,
$$

[5, p. 99], from (3.6) one obtains an expansion of a type different from formula (4.4) of [1], namely,

$$
\begin{aligned}
& \frac{1-q}{\phi\left(1,1 / 2,3 / 2, q^{2}, z^{2}\right)}=(1-q)\left[b_{0}+\frac{a_{1}}{b_{1}}+\frac{a_{2}}{b_{2}}+\ldots\right], \quad|q|<1, \\
& a_{p+1}=\frac{q^{2 p+1} z^{2}\left(1-q^{2 p+2}\right)\left(1-q^{2 p} z^{2}\right)}{1-q^{2 p+3}}, \\
& b_{p}=1-\frac{q^{2 p} z^{2}\left(1+q^{-1}-q^{2 p-1}-q^{2 p+1}\right)}{1-q^{2 p+1}}, \quad \quad p=0,1, \cdots .
\end{aligned}
$$

\section{BIBLIOGRAPHY}

1. E. Frank, A new class of continued fraction expansions for the ratios of Heine functions, Trans. Amer. Math. Soc. vol. 88 (1958) pp. 288-300.

2. - A new class of continued fraction expansions for the ratios of hypergeometric functions, Trans. Amer. Math. Soc. vol. 81 (1956) pp. 453-476.

3. E. Heine, Über die Reihe

$$
1+\frac{\left(q^{\alpha}-1\right)\left(q^{\beta}-1\right)}{(q-1)\left(q^{\gamma}-1\right)} z+\frac{\left(q^{\alpha}-1\right)\left(q^{\alpha+1}-1\right)\left(q^{\beta}-1\right)\left(q^{\beta+1}-1\right)}{(q-1)\left(q^{2}-1\right)\left(q^{\gamma}-1\right)\left(q^{\gamma+1}-1\right)} z^{2}+\cdots,
$$

Jrn. für Math. vol. 32 (1846) pp. 210-212.

4. —, Untersuchungen über die Reihe

$$
1+\frac{\left(1-q^{\alpha}\right)\left(1-q^{\beta}\right)}{(1-q)\left(1-q^{\gamma}\right)} z+\frac{\left(1-q^{\alpha}\right)\left(1-q^{\alpha+1}\right)\left(1-q^{\beta}\right)\left(1-q^{\beta+1}\right)}{(1-q)\left(1-q^{2}\right)\left(1-q^{\gamma}\right)\left(1-q^{\gamma+1}\right)} z^{2}+\cdots
$$

Jrn. für Math. vol. 34 (1847) pp. 285-328.

5. - - Handbuch der Kugelfunctionen, vol. 1, Berlin, Reimer, 1878.

6. O. Perron, Die Lehre von den Kettenbrüchen, vol. 2, 3d. ed., Stuttgart, Teubner, 1957.

UNIVERSITY OF ILLINOIS,

Chicago, Illinois 